\title{
Goal orientated Brain-Computer interfaces for Control: a virtual smart home application study
}

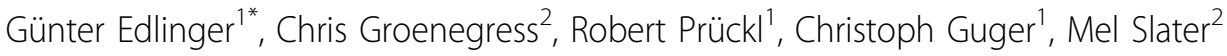 \\ From Nineteenth Annual Computational Neuroscience Meeting: CNS*2010 \\ San Antonio, TX, USA. 24-30 July 2010
}

A brain-computer interface (BCI) is a new communica-
tion channel between the human brain and a computer
without using any muscle activities. Applications of $\mathrm{BCI}$
systems comprise communication, restoration of movements or environmental control [1].

Within this study we propose a combined P300 [2] and steady state visually evoked potential (SSVEP, [3]) based BCI system for controlling a smart home in a virtual environment implementation with a high accuracy and a high degree of freedom. The system shows 20 to 45 selectable commands on a computer screen. Whenever a target command is flashing up, a P300 response can be detected from the ongoing brain activity and a control command is initiated. However, some command groups like "move right, move left, move forward, and move backward or stop" do not need an extended user interface. For such commands a SSVEP based BCI approach was implemented consisting of light sources flickering at user selectable different frequencies. The subject simply watches one of the flashlights. Specific commands are connected with the different flickering lights. The user selected command can be detected as the same flickering frequency can be found in the brain activity. A further big advantage of the implemented approach is that the so-called zero class (no command is selected) is reliably detected. Hence even a direct control of a device can be realized. If the subject does not want to control the device then he/she simply ignores the flashing lights. A total of 12 subjects participated in the P300 experiment and the mean accuracy was $79.3 \%$, $69.6 \%$ and $53.6 \%$ for 8,4 and only 2 flashes per item. The best result was achieved for subject 6 with $100 \%$ accuracy for 8 and 4 flashes. The worst results had subject 3 with only $30 \%$ accuracy for 2 flashes. A total of 3 subjects participated in the SSVEP experiment displaying a mean error rate of $3-5 \%$.

\section{Conclusion}

The combination of a P300 and SSVEP based BCI allows realizing both i) a synchronous goal orientated control system using the P300 interface satisfying the intention of the user more naturally, e.g. sending the command grasp a glass of water; here one can select a command from a large command base and ii) a reliable asynchronous more direct control of devices based on a SSVEP interface; here just a few commands can be selected with very good performance including zeroclass detection.

\section{Acknowledgements}

The work was funded by the EU projects PRESENCCIA and SM4ALL.

\section{Author details}

'g.tec, Guger Technologies, Herbersteinstrasse 60, Graz, A-8020, Austria ${ }^{2}$ Centre de Realitat Virtual (CRV), Universitat Politècnica de Catalunya, Barcelona, Spain.

\section{Published: 20 July 2010}

\section{References}

1. Wolpaw JR: Brain-computer interfaces as new brain output pathways. J. Physiol 579:613-619.

2. Krusienski DJ, Sellers EW, Cabestaing F, Bayoudh S, McFarland DJ, Vaughan TM, Wolpaw JR: A comparison of classification techniques for the P300 Speller. J.Neural Eng. 3:299-305.

3. Friman O, Volosyak I, Graser A: Multiple Channel Detection of SteadyState Visual Evoked Potentials for Brain-Computer Interfaces. Biomedical Engineering, IEEE Transactions on 2007, 54(4):742-750.

\section{doi:10.1186/1471-2202-11-S1-P134}

Cite this article as: Edlinger et al.: Goal orientated Brain-Computer interfaces for Control: a virtual smart home application study. BMC Neuroscience 2010 11(Suppl 1):P134.

\footnotetext{
* Correspondence: edlinger@gtec.at

'g.tec, Guger Technologies, Herbersteinstrasse 60, Graz, A-8020, Austria
} 